\title{
CENTRIST'S CURSE? AN ELECTORAL COMPETITION MODEL WITH CREDIBILITY CONSTRAINTS
}

\author{
SELIM JÜRGEN ERGUN \\ Middle East Technical University \\ Northern Cyprus Campus, Economics Program \\ Kalkanli (Güzelyurt), TRNC, Turkey \\ sergun@metu.edu.tr
}

Published 21 September 2015

\begin{abstract}
I analyze a model of electoral competition in which candidates' need of credibility restricts their policy choice to a subset of the policy space, their ideology set. I focus on three party competition where candidates care about winning and their share of votes. I show that centrist parties are highly disadvantaged compared to leftist and rightist ones losing the election under a wide range of parameters. I also show that centrist parties' winning opportunities increase under a run-off system.
\end{abstract}

Keywords: Electoral competition; plurality; run-off; credibility; spatial models.

JEL Classification: D72.

\section{Introduction}

In the last decades, it has been argued that the political view of society members (voters) has smoothened compared to the past and that most people would define themselves to be more centrist. A study conducted by CIS in Spain shows that the response to the question "In politics it is being talked about left and right. On a scale where 0 is left and 10 is right where would you put yourself?" had a mean of 4.56 in 1997 and 4.32 in 2005. ${ }^{1}$ More importantly, in $199751.3 \%$ of the people who answered the question located themselves between 4 and 6 , that is, in the very center of the political spectrum. Considering this aspect, one should expect that centrist parties should have encountered an increasing advantage to win elections. Daalder (1984) states that the idea of defining themselves as centrist parties has been considered attractive by politicians. Duverger (1964) argues however, that "although the "Center" is the main force of the parliamentary life", that is, although the majority of voters are located ideologically close to the very center of the political spectrum, the electoral system favors ideologically leftist or rightist parties. This paper intends to show the conditions under which one could think that Duverger's statement applies by analyzing a three candidate electoral competition model and by arguing

\footnotetext{
${ }^{1}$ The studies are Latinobarometro II and Latinobarometro VIII.
} 
that plurality rule is an electoral system that favors leftist and rightist parties whereas a centrist candidate would have more chances of winning under a run-off rule than under plurality rule.

One of the main features of my analysis is the consideration of credibility constraints. In the real world electoral competition, it is a rather rare situation that candidates can choose their policy or campaigning points among all the hypothetically possible ones. Normally, we see that candidates face restrictions over the sets of policies that they can propose as campaign promises. Furthermore, one can observe that the set of policies that are credible for one candidate usually does not coincide with the set of policies that are credible for a different candidate. In order to formalize this observation, in this paper I will assume that each candidate (or party) will be able to choose its policy point from a certain subset of the whole interval of policy points. In a one-dimensional (1D) policy space, the strategy set of a candidate, that is, the subset of the policy space from which a candidate can choose a policy point that would be considered as credible by the voters would simply be an interval of the policy line. It can be argued that, the larger the interval of a candidate, the higher is the credibility that he has among the voters.

Think about parties or candidates that have been competing for a certain number of elections. A party having claimed or acted for a long period to be ideologically leftist would lose its credibility if it were to state that from now on it were to follow a rightist point of view. Alesina (1988) states that candidates obtain credibility through reputation in an infinitely repeated game. This reputation, for a party that wins the election, makes deviations from the announced policy point to another policy which is preferred by the party too costly. This result, I believe, could be considered as a justification for the assumption that I will follow in terms of the permissible intervals. It is assumed that competing candidates have a history of past competition and the specified intervals define the limit points of policy announcements that would be considered as credible by voters.

I consider an electoral competition where candidates have a history of policy choices. This history defines them ideologically as leftist, rightist or centrist. In my framework, one could say that "Voters discipline candidates by believing some promises a candidate makes as long as" (Aragones et al., 2007, p. 848) these promises coincide with the set of promises that voters accept as credible in terms of the ideological statements of the candidates. That is, the intervals of acceptable policy points for each ideology are defined as the sets of policy points that voters consider credible given the party's history of policy choices. Aragones et al. (2007) show that in a repeated electoral competition where voters can punish candidates, parties can credibly commit to policy points which belong to an interval around their ideal points. There are also many empirical works focusing on parties' policy shifts over time. Tavits (2007), for instance, using data from 23 countries over a time span of 40 years shows that a policy shift in principled issues will lead to voter withdrawal as it is a sign of inconsistency and lack of credibility. Adams et al. (2006), on the other hand, using data from eight Western European countries over a time span of 12 years find evidence that voters penalize in terms of votes so called niche parties (extreme rightist, green communist etc.) when those parties moderate their policy positions. Using data of 14 countries, Bowler (1990) finds that parties do not change their policy positions after 
losing an election which he argues is consistent with the view that changing policies may alienate even more voters.

In the light of the empirical findings and taking the theoretical result of Aragones et al. (2007) as granted, I assume in my model that these intervals are exogenously determined considering that candidates already have a history of past choices which limits parties' ability to change their platform beyond certain limits drawn by these intervals.

I consider an electoral competition on a 1D policy space, where voters vote sincerely, with no abstention and where each candidate can choose its policy point from a given subset of the whole policy space. Candidates possess full information about the distribution of ideal points of voters. I first consider a one-round electoral system and analyze the characteristics of the equilibrium outcomes for different credibility sets for three candidate (leftist, rightist and centrist) elections. I assume that the winner is determined by plurality rule. I analyze the equilibrium outcomes, under different specifications for the intervals from which candidates can choose their policy points.

Cox (1987) shows that for a uniform distribution of voters, for candidates which are plurality maximizers and for odd numbers of candidates $(n)$ with $n \geq 3$, there exists no Nash Equilibrium in pure strategies. In fact, for three candidate competition he shows that there exist no equilibrium for a general distribution under plurality rule when candidates have three different types of utility functions. This result would also hold in my model, if candidates do not face any credibility constraints. The credibility constraints introduced in my model, however, give rise to a pure-strategy equilibrium in the cases I consider.

Regarding the main results, I show that in equilibrium the centrist candidate is highly disadvantaged compared to the other two candidates, that is, he is less likely to win. The centrist candidate loses under a wide range of parameters and even in cases where more than half of the voters' ideal point lie in his credibility set. Under a uniform distribution, for instance, a centrist candidate only wins if his interval is at least four times as large as the intervals of his opponents. In other words, the centrist candidate wins when his opponents face rather small credibility sets and when the share of voters with ideal points in his credibility set is considerably high. When the centrist candidate cannot win, in many cases his policy choice determines the winner.

Having shown that a centrist candidate faces disadvantages under plurality rule, the next step would be to argue whether this also holds for different election rules. Thus, I consider a run-off rule. Under the rule I consider, if in the first round none of the candidates reaches an absolute majority the candidates who got the first and second highest amount of votes run for a second time whereas the other candidates are eliminated. The winner is the candidate who gets the highest amount of votes in the second round, where in each round voters are required to cast a new ballot. The run-off rule I analyze is similar to the system "used for presidential elections in France and several Latin American countries and in Israel between 1996 and 2001 to elect a prime minister" (Callander, 2005). ${ }^{2}$ I show that the run-off rule can be more advantageous for centrist candidates. That is, the centrist candidate can win for larger credibility sets of his opponents compared to plurality rule.

\footnotetext{
${ }^{2}$ Callander (2005) gives also examples of other run-off rules.
} 
A somehow similar result is found by Bordignon et al. (2013). Considering both office and policy motivated parties and comparing plurality rule with run-off rule where parties can merge to run together they find that under run-off rule the equilibrium policy is less extremist.

The outline of the paper is as follows. In the next section, I build up the model. In Section 3, under the specifications of the model, I focus on the analysis of a three candidate electoral competition under plurality rule. In Section 4, I analyze the electoral competition under a run-off rule. In the last section, I conclude with a brief discussion of the results.

\section{Model}

The model that I setup follows the classical analysis of Hotelling (1929) and Downs (1957). I construct a spatial model of elections where the policy space is represented by the interval [0,1], where 0 could be considered as the most extreme leftist point and 1 as the most extreme rightist one.

\subsection{Game}

The game consists of a one period electoral competition. There are three candidates and a continuum of voters. Candidates declare their policy points simultaneously before the election and full-commitment is assumed. Each candidate can credibly commit to a policy as long as it belongs to a specified subset of the policy space. I assume that a non-credible strategy is always dominated by a credible one and therefore, I consider only credible strategies as potential equilibrium strategies. Thus, the relevant strategy set for a candidate will be defined as a particular subset of the interval. The solution concept used is Nash equilibrium. The outcome of the game is the winner of the election who is determined by plurality rule, that is, the candidate who gets the highest amount of votes is the winner, or by a run-off rule. In the case of a tie, as a tie breaking rule, I assume that each candidate sharing the highest percentage of votes will have the same probability of winning.

\subsection{Candidates}

I consider an environment in which three candidates $j$ where $j \in J$ and $J=\{L, C, R\}$ are competing. They could be considered as a leftist $(L)$, a centrist $(C)$ and a rightist $(R)$ candidate. I assume that the leftist candidate can choose a point from $[0, a]$, the centrist candidate can choose from $[b, 1-b]$ and the rightist candidate from $[1-a, 1]$ with $a \in(0,1)$ and $b \in\left(0, \frac{1}{2}\right)$. Before the election, all candidates declare their policy choices. The policy point chosen by the leftist candidate is denoted as $x_{L}$, the policy point chosen by the centrist candidate as $x_{C}$ and the one of the rightist candidate is denoted as $x_{R}$. Candidates are purely office-motivated, ${ }^{3}$ i.e., they care only about winning the election.

\footnotetext{
${ }^{3}$ In an earlier version of the paper, I considered candidates with a lexicographic utility function: Candidates primarily care about winning but also about the share of votes they get. Since they are office-motivated the utility obtained from winning outweighs the utility obtained from the share of votes. So, their aim is to win with the highest share of votes possible. A candidate who cannot win, simply chooses the policy point that maximizes his share of votes. The main results of the paper in that case remain the same. The analysis is available upon request.
} 
Thus, the utility function $\left(U_{j}\right)$ of candidates takes the following form:

$U_{j}= \begin{cases}\bar{U} & \text { if } j \text { wins the election } \\ \frac{\bar{U}}{n} & \text { if } j \text { gets the highest amount of votes with } n-1 \text { more parties }(n=2,3)\end{cases}$

where $\bar{U}$ is a fixed utility obtained from winning.

\subsection{Voters}

For the whole analysis, I assume that there is a continuum of voters who have singlepeaked preferences over the policy space with $x_{i} \in[0,1]$ referring to their ideal points for all $i \in I$. The distribution of voters' ideal points is given by a continuous distribution function $F(x)$ with $x \in[0,1]$. I assume that voters vote sincerely. As the paper focuses on credible strategies, if $x_{L}<x_{C}<x_{R}$, the voters vote for the candidate whose policy choice is closest to their ideal point. ${ }^{4}$ Moreover, I exclude the possibility of abstention by thinking of a law making voting obligatory where the penalty outweighs the cost of voting (or it could be assumed that voting has no cost). Indeed voting is compulsory in democracies such as Belgium, Australia, Greece, Luxembourg, Uruguay and others. ${ }^{5}$

For the following analysis, it will be assumed that candidates possess complete information about the distribution of the voters. Throughout the whole analysis, I denote the ideal point of the median voter as $m$.

\section{Restricted Electoral Competition Under Plurality Rule}

In this section, I analyze a three party electoral competition under plurality rule. The partition of the policy space is such that the leftist candidate can choose a policy point from the interval $[0, a]$, with $0<a<\frac{1}{2}$, the centrist candidate from $[b, 1-b]$ with $0<b<\frac{1}{2}$ and the rightist candidate from $[1-a, 1]$. In order to cover all possibilities, I analyze three cases: $a=b, b>a$ and $a>b$. The first two cases are analyzed below and the last case is analyzed in Appendix A. The figure below depicts the partition of the policy space for the first two cases:

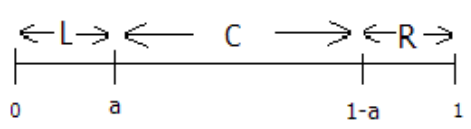

Figure 1. Partition of the Policy Space when $a=b$

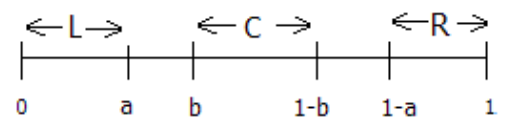

Figure 2. Partition of the Policy Space when $b>a$

\footnotetext{
${ }^{4}$ For a specification of the credibility sets analyzed in the Appendix A, this ordering of candidates may not necessarily hold. I describe voter's behavior in those cases in Appendix A.

${ }^{5}$ For more detail on countries where voting is obligatory see Chong and Olivera (2008).
} 
For certain partitions of the policy space, it is possible that two candidates are located on the same policy point. For this cases, I assume that voters behave as the following assumption suggests.

Assumption 1. If $x_{L}=x_{C}$, then all voters with $x_{i}<x_{L}$ vote for the leftist candidate while all voters with $\left(x_{R}+x_{C}\right) / 2>x_{i}>x_{L}$ vote for the centrist candidate. Similarly, if $x_{R}=x_{C}$, then all voters with $x_{i}>x_{R}$ vote for the rightist candidate while all voters with $\left(x_{L}+\right.$ $\left.x_{C}\right) / 2<x_{i}<x_{R}$ vote for the centrist candidate.

This assumption could be considered as a tie-breaking rule for the voters. Throughout the whole analysis that follows, I assume that voters behave as Assumption 1 suggests.

For three candidates, as Osborne (1995) argues, maximizing the probability of winning is not strategically equivalent to maximizing the share of votes. That is, a candidate who would win may increase his share of votes by locating himself closer to one of his opponents and thereby increasing the share of votes of his other opponent that much so that he cannot win any more. Therefore, the optimal strategy of a candidate that cares about winning under plurality does not need to coincide with the strategy that maximizes his share of votes.

Which type of equilibria would we observe for an arbitrary distribution of ideal points of voters? If the ideal point of the median voter is located in the interval of the leftist candidate, or in the interval of the rightist candidate or is located outside the intervals of all candidates but closer to the leftist or rightist one, then the results are as follows ${ }^{6}$ :

Proposition 1. Let Assumption 1 hold. Then, under an arbitrary distribution of ideal points:

Case 1. $\mathrm{a}=\mathrm{b}$ : If $m<a$, the winner is the leftist candidate and if $m>1-a$, the winner is the rightist candidate.

Case 2. $\mathrm{b}>$ a: If $m \in\left[0, \frac{a+b}{2}\right)$, the winner is the leftist candidate and if $m \in\left(1-\frac{a+b}{2}, 1\right]$ the winner is the rightist candidate.

If $b>a$, for the same value of $a$ the intervals in which the winner is the leftist or rightist candidate for sure for any distribution of ideal points of voters are larger than when $a=b$ since the centrist candidate is forced to choose from a more restricted interval than before.

Notice also that choosing $x_{L}=a$ and $x_{R}=1-a$ would maximize the vote share of the leftist and rightist candidate respectively. Moreover, for those two candidates choosing a more centrist strategy will reduce automatically the votes the centrist candidate gets. Thus, unless they loose the election for any policy choice for sure, $x_{L}=a$ and $x_{R}=1-a$ are weakly dominant strategies for the leftist and rightist candidate respectively. In what follows, as a refinement I will require each candidate to choose a weakly dominant strategy as long as such a strategy exists.

Now suppose that $m$ lies either in the interval of the centrist candidate or closer to him than to any other candidate and suppose that the distribution of ideal points of voters is represented by a continuous and increasing cumulative distribution function. Has the centrist candidate a chance to win?

\footnotetext{
${ }^{6}$ For a detailed proof of all propositions see Appendix B.
} 
Whether the centrist candidate will have a chance of winning the election or not depends both on the distribution of ideal points and on the size of its rivals' credibility sets. So, to be able to determine whether the centrist candidate is disadvantageous compared to his rivals, assume that ideal points' distribution corresponds to a Beta distribution.

The standard Beta distribution gives the probability of a value $x$ on the interval $[0,1]$, its density depends on two parameters, $\alpha$ and $\beta(\alpha, \beta>0)$ and its density has the following functional form ${ }^{7}$ :

$$
\operatorname{Beta}(\alpha, \beta)=\frac{x^{\alpha-1}(1-x)^{\beta-1}}{B(\alpha, \beta)},
$$

where $B$ is the beta function having the form of:

$$
B(\alpha, \beta)=\int_{0}^{1} t^{\alpha-1}(1-t)^{\beta-1} d t .
$$

Suppose that $a=b$ and consider unimodal symmetric distributions, i.e., $\alpha=\beta$ such that the mean and mode are equal to $\frac{1}{2} .{ }^{8}$ If Assumption 1 holds, whenever the candidates have a chance of winning, $x_{L}=a$ and $x_{R}=1-a$ are weakly dominant strategies for the leftist and rightist candidates. Moreover, since the mode is at $\frac{1}{2}$, which lies in the interval of the

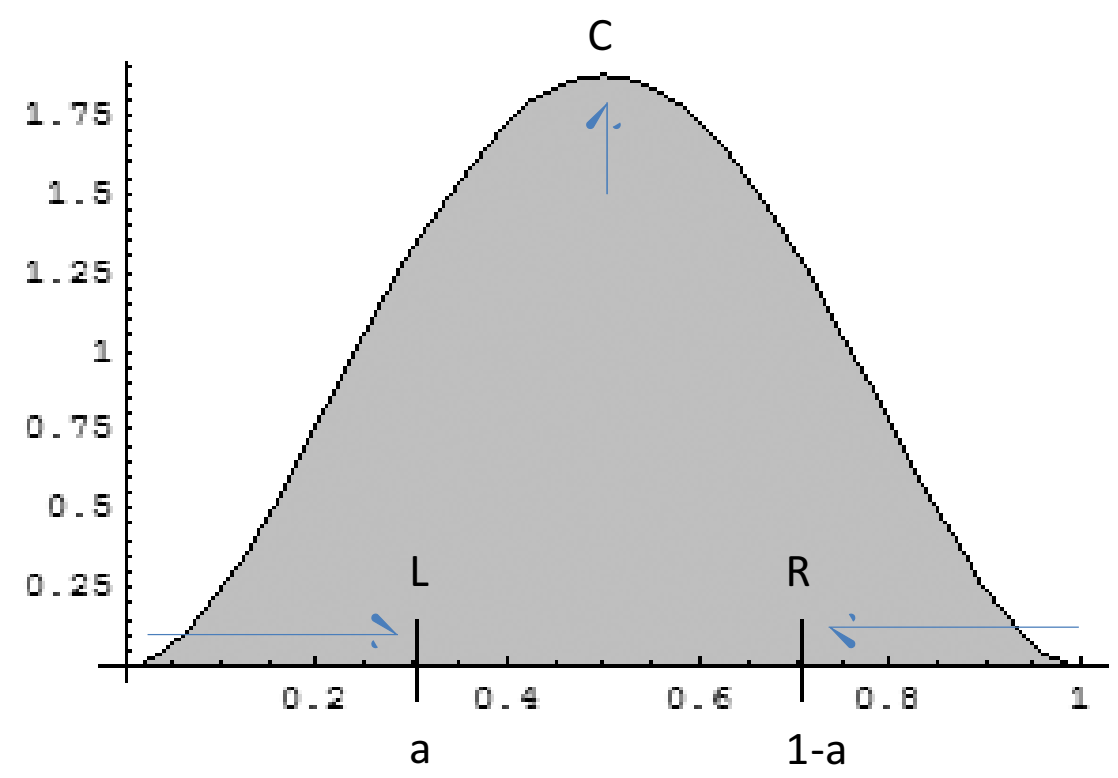

Figure 3. The Beta (3,3) Distribution and Candidates' Optimal Choices

\footnotetext{
${ }^{7}$ If $\alpha=1$ and $\beta>1$, we obtain a strictly decreasing distribution. If $\alpha>1$ and $\beta=1$, then we obtain a strictly increasing distribution. If $\alpha, \beta>1$, then the distribution is unimodal and its mode is $\frac{\alpha-1}{\alpha+\beta-2}$ and its mean is $\frac{\alpha}{\alpha+\beta}$. If $\alpha=\beta$, we have a symmetric distribution with mode and mean equal to $\frac{1}{2}$.

${ }^{8}$ Adams and Somer-Topcu (2009) graph the responses given to a Eurobarometer (2002) survey question regarding voters' self-placement on a 0 (extreme left)-10 (extreme right) left-right scale for 15 European countries. The graphs show that in most of the cases the voters' distribution is unimodal with both a heavy concentration around the center and a peak on the midpoint resembling a unimodal Beta distribution.
} 
centrist candidate, $x_{C}=\frac{1}{2}$ is a weakly dominant strategy for the centrist candidate. Figure 3 visualizes a unimodal symmetric Beta distribution and the optimum choices of the three candidates.

Therefore, in equilibrium, the share of votes of the centrist candidate $\left(s_{C}\right)$ can be calculated as follows:

$$
s_{C}=\int_{1 / 4+\frac{\alpha}{2}}^{3 / 4-\frac{\alpha}{2}} \operatorname{Beta}(\alpha, \alpha) d x .
$$

Because of symmetry, $s_{L}=s_{R}=\frac{1-s_{C}}{2}$. Therefore, for any value of $a$ (the parameter defining the size of intervals of the candidates), the values of $\alpha$ for which the centrist candidate wins the election can be determined. As $\alpha$ increases, the share of votes of the centrist candidate increases. The critical value of $\alpha$ can be found by setting the share of votes of the centrist candidate equal to $\frac{1}{3}$. For any $\alpha$ bigger than the critical value, the centrist candidate is the winner.

The $\alpha$ value that solves the above equation $\left(\alpha^{*}\right)$ gives the parameter value of the Beta distribution for which all three candidates would get the same share of votes. For any $\alpha>\alpha^{*}$, the centrist candidate wins the election, whereas for any $\alpha<\alpha^{*}$, the leftist and rightist candidates win with equal probability. For different sizes of intervals the results are as follows:

Table 1.

\begin{tabular}{lrc}
\hline Value of a & Critical $\alpha$ & Share of votes in $[\mathrm{a}, 1-\mathrm{a}]$ \\
\hline $6 / 24$ & 1.6652 & $63.76 \%$ \\
$7 / 24$ & 2.3250 & $62.84 \%$ \\
$8 / 24$ & 3.5329 & $53.81 \%$ \\
$9 / 24$ & 6.1347 & $61.68 \%$ \\
$10 / 24$ & 13.5589 & $63.76 \%$ \\
\hline
\end{tabular}

The results show that, as the interval size of the centrist candidate decreases, the critical value of $\alpha$ increases, that is the voters should be more concentrated around the center. For all interval sizes, the percentage of voters whose ideal points are in the interval of the centrist candidate is around $60 \%$. However, the centrist candidate gets roughly only the half of these votes. So, we can verify that the centrist candidate is disadvantaged compared to the other two candidates.

A special case of the Beta distribution is obtained if $\alpha=\beta=1$. In that case, we obtain the uniform distribution on the interval $[0,1]$. In what follows, to describe the whole set of equilibria, I consider such a distribution. The following proposition states the conditions for the existence of an equilibrium in which the centrist candidate wins and also describes the equilibria where the centrist candidate cannot win when $a=b$ and $b>a$ and the median voter is located outside the region described in Proposition 1. The result is as follows:

Proposition 2. Let Assumption 1 hold. Under a uniform distribution of ideal points of voters: 
(i) For $a=b$ : (a) If $a<\frac{1}{6}$ the winner is the centrist candidate and $x_{C} \in(3 a, 1-3 a)$. (b) If $a=\frac{1}{6}$, all three candidates tie and $x_{C}=\frac{1}{2}$. (c) If $a>\frac{1}{6}$, the winner is the leftist candidate if $x_{C} \in\left(\frac{1}{2}, 1-a\right]$, the rightist candidate if $x_{C} \in\left[a, \frac{1}{2}\right)$ and L and $R$ tie if $x_{C}=\frac{1}{2}$.

(ii) For $b>a$ : (a) If $a<\frac{1}{6}$ the winner is the centrist candidate and $x_{C} \in(3 a, 1-3 a)$ if $a>\frac{b}{3}$ and $x_{C} \in[b, 1-b]$ if $a \leq \frac{b}{3}$. (b) If $a=\frac{1}{6}$, all three candidates tie and $x_{C}=\frac{1}{2}$. (c) If $a>\frac{1}{6}$, the winner is the leftist candidate if $x_{C} \in\left(\frac{1}{2}, 1-b\right]$, the rightist candidate if $x_{C} \in\left[b, \frac{1}{2}\right)$ and $L$ and $R$ tie if $x_{C}=\frac{1}{2}$.

The conclusion that can be drawn is that, with intervals intersecting at a single point the centrist candidate can only win if the leftist and rightist candidates are forced to be polarized. The degree of polarization should be so large that the credibility set of the centrist candidate is at least four times as large as those of his opponents. Moreover, two thirds of all voters' ideal points are located in the centrist candidate's credibility set.

If $b>a$, the results are very similar to the case where $a=b$. The critical value, that makes the centrist candidate the winner is the same, namely, $\frac{1}{6}$. This implies that, again, for the centrist candidate to win the election his credibility set should be at least four times as large as those of his opponents. The only difference is that, if one considers the same value of $a$ for both cases, since in the case at hand $b>a$, whether he wins or not, the interval in which the centrist candidate can locate himself is smaller than it is when $a=b$ leaving him with a smaller set of choice.

\subsection{Discussion of the results}

The analysis above shows that the centrist candidate is clearly disadvantaged compared to his leftist and rightist rivals under plurality rule. The results also show that the winner depends heavily on the distribution of voters' ideal points. The leftist or rightist candidates are the winners if the median voter's ideal point is located in their credibility set and they apply the policy corresponding to the most centrist policy point allowed by their credibility sets. Under a uniform distribution of ideal points, for instance, where the median voter's ideal point is at the midpoint ( $C$ 's interval), the centrist candidate can only win if his interval is four times as large as his opponents'. In this case, for a policy choice that is not too far from his two opponents' choices, that is, for a policy choice around the middle point, the centrist candidate is the winner. A necessary condition such that the centrist candidate wins is that the median voter's ideal point belongs to the interval of the centrist candidate.

For an arbitrary distribution of ideal points, when the ideal point of the median voter is in the interval of the centrist candidate, the winner can be any of the three candidates depending on the distribution of ideal points of voters. As the previous analysis shows, it might well happen that the centrist candidate does not win even though his interval includes more than half of the voters.

The centrist candidate needs heavily concentrated ideal points of voters around the middle of the space. That is, a centrist candidate can only win if more than half of the voters' ideal points are located only in his credibility set. For some distributions, he even needs $60 \%$ or more of support in his credibility set. In short, the centrist candidate needs a 
much higher amount of voters in his credibility set compared to his opponents in order to have a chance to win the election.

Some asymmetry in the distribution of ideal points gives the centrist candidate higher chances of winning. That is, for a skewed distribution where the median voter is located at a point to the left or right of the middle point but still only in the interval of the centrist candidate, a centrist candidate can win with a less concentrated distribution in his credibility set compared to a symmetric distribution. ${ }^{9}$

One point that should be taken into account is that I only analyzed symmetric intervals. It should not be difficult to argue that, the conclusions would be almost identical under asymmetric intervals.

Of course, it should not be forgotten that these results are implied by plurality rule. Therefore, in order to see whether this disadvantage of a centrist candidate would also hold under another election rule, in the next section the same electoral competition as before will be analyzed under a run-off rule.

\section{Restricted Electoral Competition under a Run-Off Rule}

Until now, I analyzed electoral competition of a single round under plurality rule. How would the outcome change if candidates would run an electoral competition under a run-off rule? The rule I consider works as described in the introduction and I assume that candidates cannot change their policy points after the first round.

We have seen that under an electoral competition where plurality rule is applied, the centrist candidate is disadvantaged compared to his opponents. Does a run-off rule change this result? I analyze the results for voters which behave according to Assumption 1. For an arbitrary continuous distribution of ideal points of voters and any of the three different partitions of the policy space analyzed the following results would hold:

Proposition 3. Under a run-off rule where Assumption 1 holds:

(i) If $m<a$, the leftist candidate wins in the first round; if $m>1-a$, the rightist candidate wins in the first round.

(ii) If neither the leftist nor the rightist candidate wins the election in the first round, then for the centrist candidate coming second in the first round is sufficient to win the election.

(iii) The centrist candidate wins for sure under run-off rule for all parameter values for which he wins under plurality rule.

(iv) The centrist candidate (weakly) wins for a larger set of parameter values under runoff rule compared to plurality rule.

To visualize the last result above, I consider again a uniform distribution of ideal points. Both if $a=b$ or $b>a$, I showed before that for $a<\frac{1}{6}$, the centrist candidate wins for sure under a one round election system. So, for $a<\frac{1}{6}$ he would compete for sure in the second

\footnotetext{
${ }^{9}$ These results were obtained by considering different uni-modal Beta distributions.
} 
round and would win for sure under the run-off rule. Therefore, I focus on the case where $a \geq \frac{1}{6}$. The results are as follows:

Proposition 4. Let Assumption 1 hold. For a uniform distribution of voters, in equilibrium, $x_{L}=a$ and $x_{R}=1-a$ and:

(i) For $a=b$ : (a) If $\frac{1}{6} \leq a<\frac{1}{4}$ the centrist candidate wins for sure choosing $x_{C} \in$ $(a, 1-3 a)$ or $x_{C} \in(3 a, 1-a)$. (b) If $a=\frac{1}{4}$, the centrist candidate can at best win with probability $\frac{1}{4}$ by choosing $x_{C}=\frac{1}{4}$ or $x_{C}=\frac{3}{4}$. (c) If $a>\frac{1}{4}$, the leftist and rightist candidates win with equal probability.

(ii) For $b>a$ : (a) If $\frac{1}{6} \leq a<\frac{1}{4}$ and $1-3 a>b$ the centrist candidate wins for sure choosing $x_{C} \in[b, 1-3 a)$ or $x_{C} \in(3 a, 1-b]$. (b) If $\frac{1}{6} \leq a<\frac{1}{4}$ and $1-3 a=b$, the centrist candidate can win by tieing once choosing $x_{C}=b$ or $x_{C}=1-b$. (c) If $\frac{1}{6} \leq a<\frac{1}{4}$ and $1-3 a<b$, the leftist and rightist candidates win with equal probability. (d) If $a \geq \frac{1}{4}$, the leftist and rightist candidates win with equal probability.

For $a=b$, if we compare the results of the run-off rule with the results of Section 3, it can be said that the centrist candidate can win for a higher range of $a$ as under plurality rule he wins for sure for $a<\frac{1}{6}$ and under run-off rule for $a<\frac{1}{4}$. Now, his interval has to be at least twice as large as those of his opponents to win rather than four times as it has to be under plurality rule.

For $b>a$, it can easily be concluded that the centrist candidate can win for a wider set of parameter values than in a one-round election as under plurality rule he could only win for $a<\frac{1}{6}$ while under run-off rule he can also win for $\frac{1}{6}<a<\frac{1}{4}$ if $1-3 a>b$ holds.

To compare the results of the two cases analyzed above, consider intervals of the same length for leftist and rightist candidates for the case where $b=a$ and for the case where $b>a$ with $\frac{1}{4}>a>\frac{1}{6}$. In order to get to the second round, the policy choice of the centrist candidate has to be located close enough to at least one of his opponents. However, if $b>a$, he might not locate himself close enough to one of his opponents if $b$ is too large (i.e., if his interval too small), while for $b=a$ this would not occur since the intervals of the leftist and centrist candidates and the intervals of the rightist and centrist candidates intersect. Therefore, if $b>a$, the centrist candidate does not necessarily win under a runoff system if $\frac{1}{4}>a$.

Moreover, in the cases where the centrist candidate can never win, different from before, under run-off rule his policy point choice does not affect the outcome. For any choice of him, the other two candidates win with equal probability.

The case where $\frac{1}{2}>a>b$ is again analyzed in Appendix A. As before, I only analyzed symmetric intervals. It should not be difficult to argue that, the conclusions would be almost identical under asymmetric intervals.

The results show that the run-off rule reduces a centrist candidate's disadvantage but it does not eliminate it. Take as an example the French presidential elections where the electoral rule is a run-off system. As stated in the Taipei Times (March 22, 2011) ${ }^{10}$ "Only

${ }_{10}$ An article written by $\mathrm{Hu}$ Tsu-Ching. 
once during the 50 years of the current French Fifth Republic did a more centrist political party win a presidential election, with Valery Giscard d'Estaing, who stayed in power for seven years. The rest of the time, presidential elections have either been won by the major left-wing, or, more often, the right-wing party." On the other hand, assuming that the vote shares of the candidates would not change by a very large amount if the electoral rule were switched from run-off to plurality, it can easily be argued that Valery Giscard d'Estaing would have lost the election under plurality as he came second (obtaining $32.6 \%$ of the votes) in the first round behind François Mitterrand who obtained $43.2 \%$ of the votes. ${ }^{11}$

\section{Conclusion}

The model I have analyzed uses the credibility that voters assign to candidates to introduce restrictions on the candidates' strategies. I assume that exogenously given restrictions about the policy space specify the policy points that candidates can credibly propose. This limitation can prevent full convergence in terms of candidates' policy choices.

The results imply that in many cases, although a majority of voters could be considered as ideologically centrist, centrist candidates have no chance of winning. This conclusion verifies Duverger's statement mentioned before up to some point. It is not uniquely the plurality rule that leads to outcomes where only two candidates get a considerable amount of votes; credibility of candidates plays also an important role. Since candidates are assumed to be office-motivated, leftist and rightist candidates have incentives to converge to each other as much as their intervals allow. The centrist candidate prefers to be as close as possible to one of his opponents for more asymmetric distributions of ideal points of voters, whereas for symmetric distributions he prefers to be as far as possible from all his opponents. The centrist candidate is better-off the larger is his interval and the smaller the interval of his opponents.

As it was discussed before, it can be said that the larger the interval of a candidate, the higher is his credibility. Therefore, it can be concluded that as the credibility of a candidate increases, he enjoys a better prospect of winning. However, for a centrist candidate to be able to win his credibility set should be much larger than those of leftist and rightist ones. Under a uniform distribution for instance, the interval of the centrist candidate should be at least four times as large as his opponents.

It can easily be argued that not only the distribution of voters but also the election rule determines the winner in the model analyzed in this paper. The model shows that leftist and rightist candidates would prefer a one-round electoral competition (plurality rule) whereas a centrist candidate would be better off under a run-off rule. That is, the election rule might help to obtain more centrist outcomes. In fact, coming second in the first round (given that no candidate obtains an absolute majority in the first round) is sufficient for a centrist candidate to win the election under a run-off rule. Broadly speaking, the electoral competition in UK can be considered as a three party competition where plurality rule applies. As Taagepera and Shugart (1989) state British Liberals favored changes in the electoral

\footnotetext{
${ }^{11}$ Data retrieved from http://www.electionresources.org/ by Manuel Alvarez-Rivera.
} 
rule which continues up today, as the plurality rule favors the two parties (Labor and Conservatives) on the left and right wings, whereas these two parties are quite happy with the system. In the last 25 years, although the majority of the voters could be considered centrist, $^{12}$ the winning party has always been either Labor or the Conservatives. The Liberal Democrats came consistently third in the run.

If the intervals of credible policies of the leftist and rightist candidates intersect, a centrist candidate cannot get any vote. Therefore, one should expect that in countries where leftist and rightist parties have high credibility, the electoral competition would take place between two parties. Centrist parties would arise when there is a policy interval that cannot be reached by any leftist or rightist party. The chance of winning of a centrist candidate would increase with the size of his interval and with a decrease in the size of the intervals of his opponents.

One important assumption maintained during the analysis is that there is no abstention. One further step would be to discard this assumption and think that a voter would not vote for any candidate if they are too far away from his ideal point. In this case, we could reach equilibria in which leftist and rightist candidates might diverge more than they do without abstention as they would have two conflicting incentives, namely, being close to the centrist candidate to "steal" some of his votes and secondly not to loose rather radical voters.

The model at hand considers exogenous credibility constraints assumed to be imposed by the voters. However, refraining from announcing a policy beyond a certain ideological limit might also be self-imposed by the candidates. That is, candidates might have a preference over their policy announcement and they might suffer a utility loss if they announce a policy different than their ideal policy. In that case, candidates would be both office- and to some extent policy-motivated and the credibility constraint would be endogenized. This would be an interesting enrichment of the current model allowing also to test whether the "Centrist's Curse" would be as strong as under the current specification.

\section{Appendix A. Intervals Overlapping with Neighbors $\left(b<a<\frac{1}{2}\right)$}

Plurality Rule: In the case at hand, the centrist candidate can locate himself to the left of the leftist candidate or to the right of the rightist candidate. For such a choice, he could not be considered as the centrist candidate anymore being more leftist than the leftist or more rightist than the rightist candidate. Therefore, the following assumption will be made:

Assumption 2. If $x_{C}<x_{L}$ or $x_{C}>x_{R}$, then the centrist candidate gets no votes.

This assumption states that voters punish the centrist candidate by giving him no votes if he chooses to be more leftist or more rightist than the corresponding candidate. That is, although voters consider some policy choices as credible claims proposed by two different candidates, they still force candidates to locate themselves according to their ideological labels. I assume for the whole section that voters behave as Assumption 2 suggests. Without this assumption, there would exist no equilibrium for a uniform distribution of

\footnotetext{
${ }^{12}$ The European Election Study (2009) results show that $55 \%$ of those who responded to the question of left-right self replacement on a scale from 0 to 10, 0 being the most leftist and 10 the most rightist, located themselves between 4 and 6 . Adams and Somer-Topcu (2009) depict a similar result using the Eurobarometer (2002) survey.
} 
ideal points of voters if $a>\frac{1}{4} \cdot{ }^{13}$ For an arbitrary distribution of ideal points of voters, if the ideal point of the median voter is located in the interval $[0, a)$, or in the interval $(1-a, 1]$, the following result is obtained:

Proposition 5. Let Assumptions 1 and 2 hold. For an arbitrary distribution of ideal points under plurality rule:

(i) If $m \in[0, a)$, L wins.

(ii) If $m \in(1-a, 1], R$ wins.

Proof. For the first case $x_{L}=a$ is a weakly dominant strategy for $L$ and for the second case $x_{R}=1-a$ is a weakly dominant strategy for $R$. Thus, the analysis becomes identical to Proposition 2.

If the location of the median voter is in the interval $[a, 1-a]$, then the winner would depend on the distribution of the ideal points and the length of the intervals of the leftist and rightist candidates. Now to be able to say more about what happens if $m$ is in this interval, consider a uniform distribution of ideal points. In this case, we get the following result:

Proposition 6. Let Assumptions 1 and 2 hold. For a uniform distribution of ideal points under plurality rule:

(i) If $a<\frac{1}{6}, x_{C} \in(3 a, 1-3 a)$ and the centrist candidate is the winner.

(ii) If $a=\frac{1}{6}$, then $x_{C}=\frac{1}{2}$ and all three candidates win with equal probabilities.

(iii) If $a>\frac{1}{6}$ and $x_{C} \in\left[a, \frac{1}{2}\right)$, the winner is the rightist candidate.

(iv) If $a>\frac{1}{6}$ and $x_{C} \in\left(\frac{1}{2}, 1-a\right]$, the winner is the leftist candidate.

(v) If $a>\frac{1}{6}$ and $x_{C}=\frac{1}{2}$ the leftist and rightist candidates win with equal probability.

Proof. Since $x_{L}=a$ and $x_{R}=1-a$ are weakly dominant strategies, $s_{C}=0$ if $x_{C}<a$ or $x_{C}>1-a$. So, in equilibrium, $x_{C} \in[a, 1-a]$. Therefore, $s_{C}=\frac{1-2 a}{2}$. Thus, the analysis becomes identical to Proposition 2(i).

The conclusion that can be drawn from the above result is that if the centrist candidate is required to locate himself to the right of the leftist candidate and to the left of the rightist candidate, the results are the same whether the intervals overlap or only intersect at a single point. Moreover, the interval of the centrist candidate does not play a role. The only parameter that affects the outcome is, $a$, the one that specifies the intervals of the leftist and rightist candidates.

Assumption 2 is strong in the sense that it states that voters would punish the centrist candidate and not the leftist or rightist one when the centrist candidate does not choose a policy point that lies between the policy points of the other two candidates. However, it can be shown that the parameter values for which the centrist candidate could win the election would be the same if voters would punish the leftist or rightist candidate rather than the centrist whenever the situation described in Assumption 2 occurs. ${ }^{14}$

\footnotetext{
${ }^{13} \mathrm{~A}$ formal analysis available upon request.

${ }^{14} \mathrm{~A}$ formal analysis available upon request.
} 
Run-off Rule: For $\frac{1}{2}>a>b$, the centrist candidate wins under a one-round electoral system only if $a<\frac{1}{6}$. So, for $a<\frac{1}{6}$ he would compete for sure in the second round and would win for sure under run-off rule as he would be located closer to the median voter compared to his opponent. Therefore, as before, I consider only the case where $a \geq \frac{1}{6}$. The result is as follows:

Proposition 7. Let Assumptions 1 and 2 hold. For a uniform distribution of voters under run-off rule:

(i) If $\frac{1}{6} \leq a<\frac{1}{4}$ the centrist candidate wins for sure choosing $x_{C} \in(a, 1-3 a)$ or $x_{C} \in(3 a, 1-a)$.

(ii) If $a=\frac{1}{4}$, the centrist candidate can win with probability $\frac{1}{4}$ by choosing $x_{C}=\frac{1}{4}$ or $x_{C}=\frac{3}{4}$.

(iii) If $a>\frac{1}{4}$, the leftist and rightist candidates win with equal probability.

Proof. As $x_{L}=a$ and $x_{R}=1-a$ are weakly dominant strategies for $L$ and $R$, respectively, $C$ never chooses $x_{C}<a$ or $x_{C}>1-a$ since he would get no votes for such a choice. So, none of the candidates can get more than half of the votes in the first round for any $a$. So, the analysis becomes identical to Proposition 4(i).

Once again, under a run-off rule the centrist candidate can win under a higher range of parameters compared to plurality rule. (for $a<\frac{1}{6}$ under plurality and for $a<\frac{1}{4}$ under runoff) As in the one-round case the parameter $b$ plays no role. The only important parameter is $a$, the one defining the length of the intervals of the leftist and rightist candidates.

\section{Appendix B}

Proof of Proposition 1. Case 1. If $m \in[0, a), L$ is the winner because for any $x_{L} \in(m, a], s_{L}>\frac{1}{2}$ which implies that $s_{L}>s_{j}$ for $j \in\{R, C\}$. If $m \in(1-a, 1], R$ is the winner because for $x_{R} \in[1-a, m], s_{R}>\frac{1}{2}$ which implies that $s_{R}>s_{j}$ for $j \in\{L, C\}$. Case 2. If $m \in\left[0, \frac{a+b}{2}\right), L$ wins because for $x_{L} \in(m, a], s_{L}>\frac{1}{2}$ which implies that $s_{L}>s_{j}$ for $j \in\{R, C\}$. If $m \in\left(1-\frac{a+b}{2}, 1\right], R$ wins because for $x_{R} \in[1-a, m), s_{R}>\frac{1}{2}$ which implies that $s_{R}>s_{j}$ for $j \in\{L, C\}$.

Proof of Proposition 2. As long as they have a possibility of winning, $x_{L}=a$ and $x_{R}=1-a$ are weakly dominant strategies for $L$ and $R$ respectively. Let's take $x_{L}=a$ and $x_{R}=1-a$ to find the critical value of $a$ for which $C$ starts winning the election. (i). In that case, $s_{C}=\frac{1-2 a}{2}$. (a) If $x_{C} \in\left(\frac{1}{2}, 1-a\right], s_{L}>s_{R}$. Therefore, $C$ wins if $s_{C}=\frac{1-2 a}{2}>s_{L}=$ $\frac{x_{C}+a}{2}$ i.e., if $x_{C}<1-3 a$. If $x_{C} \in\left[a, \frac{1}{2}\right), s_{R}>s_{L}$. Therefore, $C$ wins if $s_{C}=\frac{1-2 a}{2}>s_{R}=$ $a+\frac{1-a-x_{C}}{2}$ i.e., if $x_{C}>3 a$. So, $C$ wins for sure choosing $x_{C} \in(3 a, 1-3 a)$ which can only be a valid interval if $a<\frac{1}{6}$ meaning that $C$ wins for sure if $a<\frac{1}{6}$. (b) For $a=\frac{1}{6}$, the above inequalities hold only for the case of equality leaving $x_{C}=\frac{1}{2}$ the only choice for $C$, resulting in equal shares of votes for all three candidates. (c) Notice that if $a>\frac{1}{6}, C$ can never win. Therefore, if $x_{C} \in\left[a, \frac{1}{2}\right)$ then $s_{R}=1-\frac{\left(1-a+x_{C}\right)}{2}>\frac{\left(a+x_{C}\right)}{2}=s_{L}$ and $R$ wins; if $x_{C} \in\left(\frac{1}{2}, 1-a\right], 1-\frac{\left(1-a+x_{C}\right)}{2}<\frac{\left(a+x_{C}\right)}{2}$ and $L$ wins; if $x_{C}=\frac{1}{2}, s_{L}=s_{R}=\frac{1-2 a}{2}$. From the tie-breaking rule, $L$ and $R$ win with equal probability. (ii) Using the same argument as 
before, $s_{C}=\frac{1-2 a}{2}$. (a) If $C$ locates himself to the right of $\frac{1}{2}, s_{L}>s_{R}$. Therefore, $C$ wins if $s_{C}=\frac{1-2 a}{2}>s_{L}=a+\frac{x_{C}-a}{2}$ i.e., if $x_{C}<1-3 a$. If he locates himself to the left of $\frac{1}{2}$, $s_{R}>s_{L}$. Therefore, $C$ wins if $s_{C}=\frac{1-2 a}{2}>s_{R}=a+\frac{1-a-x_{C}}{2}$ i.e., if $x_{C}>3 a$. $(3 a, 1-3 a)$ is a valid interval only if $a<\frac{1}{6}$. Notice that if $1-3 a>1-b$ i.e., if $\frac{b}{3}>a$, then $1-3 a$ and $3 a$ lie outside the interval of $C$. So, if $a<\frac{1}{6}$ and $a>\frac{b}{3}, C$ wins for sure with $x_{C} \in(3 a, 1-3 a)$. If $a<\frac{1}{6}$ and $\frac{b}{3} \geq a, C$ wins for sure with $x_{C} \in[b, 1-b]$. (b) Clearly, for $a=\frac{1}{6}$, the above inequalities hold only for the case of equality leaving $x_{C}=\frac{1}{2}$ as the only choice for $C$, resulting in equal shares of votes for all candidates. (c) If $a>\frac{1}{6}, C$ cannot win. So, if $x_{C}=\frac{1}{2}$, then $s_{L}=s_{R}=\frac{2 a+1}{4}>\frac{1-2 a}{2}=s_{C}$. From the tie-breaking rule, $L$ and $R$ win with equal probability. If $x_{C} \in\left(\frac{1}{2}, 1-b\right], s_{L}>s_{R}$ and $L$ wins. If $x_{C} \in\left[b, \frac{1}{2}\right)$ then $s_{R}>s_{L}$ and $R$ wins.

Proof of Proposition 3. (i) If $m<a(m>1-a), s_{L}>0.5\left(s_{R}>0.5\right)$ for $x_{L}=a$ $\left(x_{R}=1-a\right)$. Thus, the winner is determined in the first round. (ii) In this case, $m$ is located in the interval of $C$. Claim. If $C$ qualifies for the second round he will for sure be closer to $m$ than his opponent in this round. Why? Because if it weren't the case, that is, if his opponent in the second round is closer to $m$, this opponent would already have acquired more than half of the votes in the first round. (iii) If $C$ wins under plurality rule for a given policy choice then he would also win the first round under the run-off rule for the same policy choice as the equilibrium policy choice of $L$ and $R$ is the same under both rules. We know that if $C$ wins under plurality rule he is necessarily closer to $m$ compared to his two opponents. So, for the same policy choice $C$ would win for sure the second round. (iv) For the plurality rule there exists a critical value of $a$ beyond which $C$ can never win. Take an $a$ slightly larger $\left(a^{\prime}=a+\varepsilon\right)$. We know that $C$ would win for sure if he gets to the second round. So, the only possibility that he does not win is that he becomes third for any policy choice in the first round. However, if $a \geq b$, due to the continuity of the distribution function, this will not happen for $\varepsilon$ sufficiently small. If $b>a$, then if $b$ is sufficiently large, examples can be found for which $C$ can never win if $a$ is only slightly higher than the critical value. $^{15}$

Proof of Proposition 4. To find the critical value of $a$ for which $C$ starts winning the election use the same type of analysis as in Proposition 2. (i) Since $x_{L}=a$ and $x_{R}=1-a$ are weakly dominant strategies for $L$ and $R$ respectively, $L$ can get at most half of the votes, which happens when $x_{C}=1-a$. Similarly, $R$ can get at most half of the votes, which happens when $x_{C}=a$. $s_{C}=\frac{1-2 a}{2}<\frac{1}{2}$ since $a \geq \frac{1}{6}$. So, none of the candidates can get more than half of the votes in the first round for any $a$. (a) Suppose that, $C$ locates himself to the left of $\frac{1}{2}$. Then, in order to get to the second round he has to get more votes than $L$ i.e., $\frac{1-2 a}{2}>a+\frac{x_{C}-a}{2}$ should be satisfied which holds for $x_{C}<1-3 a$. If $C$ locates himself to the right of $\frac{1}{2}$, then, in order to get to the second round he has at least to get more votes than $R$ i.e., $\frac{1-2 a}{2}>a+\frac{1-a-x_{C}}{2}$ should be satisfied which holds for $x_{C}>3 a$. So, $C$ should choose $x_{C} \in[a, 1-3 a)$ or $x_{C} \in(3 a, 1-a]$ to get to the second round. Clearly, it can only be satisfied if $a<\frac{1}{4}$ and these two intervals do not intersect for $\frac{1}{6} \leq a$. Choosing $x_{C}=a$ or

15 Available upon request. 
$x_{C}=1-a, C$ would get in the second round the same amount of votes as his opponent ( $R$ and $L$ respectively). Therefore, choosing $x_{C} \in(a, 1-3 a)$ or $x_{C} \in(3 a, 1-a), C$ wins the second round for sure. (b) If $a=\frac{1}{4}$, then $a=1-3 a$, so to have the chance to get to the second round after the tie-braking, $C$ has to choose $x_{C}=\frac{1}{4}$ or $x_{C}=\frac{3}{4}$. Doing so, he gets the same share of votes as his opponent in the second round meaning that $C$ could win after two ties. (c) If $a>\frac{1}{4}$, then for $x_{L}=a$ and $x_{R}=1-a, s_{j}>s_{C} j \in\{L, R\} \forall x_{C} \in[a, 1-a]$. So, $L$ and $R$ compete in the second round and win with equal probability getting the same share of votes. (ii) For a similar reasoning as in part $i$, none of the candidates can get more than half of the votes in the first round for any $a$. (a) Suppose that, $C$ locates himself to the left of $\frac{1}{2}$. Then, in order to get to the second round he has to get more votes than $L$ i.e., $\frac{1-2 a}{2}>a+\frac{x_{C}-a}{2}$ should be satisfied which holds for $x_{C}<1-3 a$. If $C$ locates himself to the right of $\frac{1}{2}$, then, in order to get to the second round he has to get more votes than $R$ i.e., $\frac{1-2 a}{2}>a+\frac{1-a-x_{C}}{2}$ should be satisfied which holds for $x_{C}>3 a$. So, $C$ should choose $x_{C} \in[b, 1-3 a)$ or $x_{C} \in(3 a, 1-b]$ to get to the second round. These are valid intervals only if $1-3 a>b$, which could only hold if $a<\frac{1}{4}$ since $b>a$ should be satisfied. These two intervals do not intersect for $\frac{1}{6} \leq a$. So, if $\frac{1}{6} \leq a<\frac{1}{4}$ and $1-3 a>b, C$ gets to the second round and wins the election. (b) If $\frac{1}{6} \leq a<\frac{1}{4}$ and $1-3 a=b$, then $C$ can only choose $x_{C}=b$ or $x_{C}=1-b$. So, he would get the same share of votes as $L$ or $R$ respectively and could get to the second round by tieing. He wins the second round for sure. (c) If $\frac{1}{6} \leq a<\frac{1}{4}$ and $1-3 a<b$, then $C$ cannot pass the first round since both $L$ and $R$ get more votes than $C$. $L$ and $R$ pass to the second round and tie in the second round getting the same share of votes. (d) The same argument as in part (c) holds.

\section{Acknowledgments}

I am grateful for the help and valuable comments of Enriqueta Aragones. I would also like to thank Oriol Carbonell and Josep Colomer for their comments.

\section{References}

Adams, J, M Clark, L Ezrow and G Glasgow (2006). Are Niche parties fundamentally different from mainstream parties? The causes and the electoral consequences of western European parties' policy shifts, 1976-1998. American Journal of Political Science, 50(3), 513-529.

Adams, J and Z Somer-Topcu (2009). Moderate now, win votes later: The electoral consequences of parties' policy shifts in 25 postwar democracies. The Journal of Politics, 71(2), 678-692.

Alesina, A (1988). Credibility and policy convergence in a two-party system with rational voters. American Economic Review, 78, 796-805.

Álvarez-Rivera, M (2014). Election resources on the internet. Available at http://www.electionresources.org/. (accessed on: March 7, 2014).

Aragones, E, TR Palfrey and A Postlewaite (2007). Political reputations and campaign promises. Journal of the European Economic Association, 5, 846-884.

Bordignon, M, T Nannicini and G Tabellini (2013). Moderating political extremism: Single vs runoff elections under plurality rule. IZA Discussion Papers 7561, Institute for the Study of Labor (IZA). 
Bowler, S (1990). Voter perceptions and party strategies: An empirical approach. Comparative Politics, 23(1), 61-83.

Callander, S (2005). Duverger's hypothesis, the run-off rule, and electoral competition. Political Analysis, 13, 209-232.

Chong, A and M Olivera (2008). Does compulsory voting help equalize incomes? Economics \& Politics, 20, 391-415.

Cox, G (1987). Electoral equilibrium under alternative voting institutions. American Journal of Political Science, 31, 82-108.

Daalder, H (1984). In search of the center of european party systems. The American Political Science Review, 78, 92-109.

Downs, A (1957). An Economic Theory of Democracy. New York: Harper and Row.

Duverger, M (1964). Political Parties: Their Organization and Activity in the Modern State London: Methuen \& Co. Ltd.

Egmond, MH Van, W Van der Brug, SB Hobolt, MN Franklin and EV Sapir (2013). European Parliament Election Study 2009, Voter Study. GESIS Data Archive, Cologne. ZA5055 Data file Version 1.1.0, doi:10.4232/1.11760.

Hotelling, H (1929). Stability in competition. Economic Journal, 39, 41-57.

Latinobarometro II by Centro de Investigaciones Sociologicas, December 1997.

Latinobarometro VIII by Centro de Investigaciones Sociologicas, October 2005.

Osborne, MJ (1995). Spatial models of political competition under plurality rule: A survey of some explanations of the number of candidates and the positions they take. The Canadian Journal of Economics, 28, 261-301.

Taagepera, R and MS Shugart (1989). Seats \& Votes. New Haven and London: Yale University Press.

Tavits, M (2007). Principle vs. pragmatism: Policy shifts and political competition. American Journal of Political Science, 51(1), 151-165.

Tsu-Ching, Hu (2011). The power of median voters to decide polls. Taipei Times. Available at http://www.taipeitimes.com/News/editorials/archives/2011/03/22/2003498781, (accessed on: February 21, 2014). 